\title{
Introduction to the AdS/CFT Correspondence
}

\author{
Igor R. Klebanov \\ Joseph Henry Laboratories \\ Princeton University \\ Princeton, New Jersey 08544, USA
}

ABstRaCT: This is an introductory review of the AdS/CFT correspondence and of the ideas that led to its formulation. We show how comparison of stacks of D3-branes with corresponding supergravity solutions leads to dualities between conformal large $N$ gauge theories in 4 dimensions and string backgrounds of the form $A d S_{5} \times X_{5}$ where $X_{5}$ is an Einstein manifold. The gauge invariant chiral operators of the field theory are in one-to-one correspondence with the supergravity modes, and their correlation functions at strong coupling are determined by the dependence of the supergravity action on AdS boundary conditions. The simplest case is when $X_{5}$ is a 5 -sphere and the dual gauge theory is the $\mathcal{N}=4$ supersymmetric $S U(N)$ Yang-Mills theory. We also discuss D3-branes on the conifold corresponding to $X_{5}$ being a coset space $T^{1,1}=(S U(2) \times S U(2)) / U(1)$. This background is dual to a certain $\mathcal{N}=1$ superconformal field theory with gauge group $S U(N) \times S U(N)$.

\section{Introduction}

String theory originated from attempts to understand the strong interactions [1]. However, after the emergence of QCD as the theory of hadrons, the dominant theme of string research shifted to the Planck scale domain of quantum gravity [2]. Although in hadron physics one routinely hears about flux tubes and the string tension, many particle theorists gave up hope that string theory might lead to an exact description of the strong interactions. Now, however, for the first time we can say with confidence that at least some strongly coupled gauge theories have a dual description in terms of strings. Let me emphasize that one is not talking here about effective strings that give an approximate qualitative description of the QCD flux tubes, but rather about an exact duality. At weak coupling a convenient description of the theory involves conventional perturbative methods; at strong coupling, where such methods are intractable, the dual string description simplifies and gives exact information about the theory. The best established examples of this duality are (super)conformal gauge theories where the so-called AdS/CFT correspondence [3,
$4,5]$ has allowed for many calculations at strong coupling to be performed with ease. In these notes I describe, from my own personal perspective, some of the ideas that led to the formulation of the AdS/CFT correspondence. For the sake of brevity I will mainly discuss the $\mathrm{AdS}_{5} / \mathrm{CFT}_{4}$ case which is most directly related to 4 -dimensional gauge theories.

It has long been believed that the best hope for a string description of non-Abelian gauge theories lies in the 't Hooft large $N$ limit. A quarter of a century ago 't Hooft proposed to generalize the $S U(3)$ gauge group of QCD to $S U(N)$, and to take the large $N$ limit while keeping $g_{\mathrm{YM}}^{2} N$ fixed [6]. In this limit each Feynman graph carries a topological factor $N^{\chi}$, where $\chi$ is the Euler characteristic of the graph. Thus, the sum over graphs of a given topology can perhaps be thought of as a sum over world sheets of a hypothetical "QCD string." Since the spheres (string tree diagrams) are weighted by $N^{2}$, the tori (string one-loop diagrams) - by $N^{0}$, etc., we find that the closed string coupling constant is of order $N^{-1}$. Thus, the advantage of taking $N$ to be large is that we find a weakly coupled string theory. It is not clear, however, how to describe 
this string theory in elementary terms (by a 2dimensional world sheet action, for example). This is clearly an important problem: the free closed string spectrum is just the large $N$ spectrum of glueballs. If the quarks are included, then we also find open strings describing the mesons. Thus, if methods are developed for calculating these spectra, and it is found that they are discrete, then this provides an elegant explanation of confinement. Furthermore, the $1 / N$ corrections correspond to perturbative string corrections.

Many years of effort, and many good ideas, were invested into the search for an exact gauge field/string duality [7]. One class of ideas, exploiting the similarity of the large $N$ loop equation with the string Schroedinger equation, eventually led to the following fascinating speculation [8]: one should not look for the QCD string in four dimensions, but rather in five, with the fifth dimension akin to the Liouville dimension of non-critical string theory [9]. This leads to a picture where the QCD string is described by a two-dimensional world sheet sigma model with a curved non-compact 5-dimensional target space. The difficult question is: precisely which target spaces are relevant to gauge theories? Luckily, we now do have answers to this question for a variety of conformal large $N$ gauge models. In these examples of the gauge field/string duality the strings propagate in 5 compact dimensions in addition to the 5 non-compact ones. In fact, these "gauge strings" are none other than type IIB superstrings propagating in curved 10-dimensional backgrounds of the form $A d S_{5} \times X_{5}$. The $A d S_{5}$ factor present in the dual description of all conformal field theories is the 5-dimensional Anti-de Sitter space which has constant negative curvature. $X_{5}$ is a compact positively curved space which depends on the specific model: the simplest example is when $X_{5}$ is a 5 -sphere leading to the dual formulation of the $\mathcal{N}=4$ supersymmetric Yang-Mills theory $[3,4,5]$ but other, more intricate, dualities have also been constructed [10, $11,12]$. The route that leads to these results involves an unexpected detour via black holes and Dirichlet branes. We turn to these subjects next.

\section{D-branes vs. Black Holes and $p$ - branes}

A few years ago it became clear that, in addition to strings, superstring theory contains solitonlike "membranes" of various internal dimensionalities called Dirichlet branes (or D-branes) [13]. A Dirichlet $p$-brane (or $\mathrm{D} p$-brane) is a $p+1 \mathrm{di}$ mensional hyperplane in $9+1$ dimensional spacetime where strings are allowed to end, even in theories where all strings are closed in the bulk of space-time. In some ways a D-brane is like a topological defect: when a closed string touches it, it can open open up and turn into an open string whose ends are free to move along the Dbrane. For the end-points of such a string the $p+1$ longitudinal coordinates satisfy the conventional free (Neumann) boundary conditions, while the $9-p$ coordinates transverse to the $\mathrm{D} p$ brane have the fixed (Dirichlet) boundary conditions; hence the origin of the term "Dirichlet brane." In a seminal paper [14] Polchinski showed that the Dp-brane is a BPS saturated object which preserves $1 / 2$ of the bulk supersymmetries and carries an elementary unit of charge with respect to the $p+1$ form gauge potential from the Ramond-Ramond sector of type II superstring. The existence of BPS objects carrying such charges is required by non-perturbative string dualities [15]. A striking feature of the D-brane formalism is that it provides a concrete (and very simple) embedding of such objects into perturbative string theory.

Another fascinating feature of the D-branes is that they naturally realize gauge theories on their world volume. The massless spectrum of open strings living on a $\mathrm{D} p$-brane is that of a maximally supersymmetric $U(1)$ gauge theory in $p+1$ dimensions. The $9-p$ massless scalar fields present in this supermultiplet are the expected Goldstone modes associated with the transverse oscillations of the $\mathrm{D} p$-brane, while the photons and fermions may be thought of as providing the unique supersymmetric completion. If we consider $N$ parallel D-branes, then there are $N^{2}$ different species of open strings because they can begin and end on any of the D-branes. $N^{2}$ is the dimension of the adjoint representation of $U(N)$, and indeed we find the maximally supersymmet- 
ric $U(N)$ gauge theory in this setting [16]. The relative separations of the $\mathrm{D} p$-branes in the $9-p$ transverse dimensions are determined by the expectation values of the scalar fields. We will be primarily interested in the case where all scalar expectation values vanish, so that the $N \mathrm{D} p$ branes are stacked on top of each other. If $N$ is large, then this stack is a heavy object embedded into a theory of closed strings which contains gravity. Naturally, this macroscopic object will curve space: it may be described by some classical metric and other background fields including the Ramond-Ramond $p+1$ form potential. Thus, we have two very different descriptions of the stack of D p-branes: one in terms of the $U(N)$ supersymmetric gauge theory on its world volume, and the other in terms of the classical RamondRamond charged $p$-brane background of the type II closed superstring theory. The relation between these two descriptions is at the heart of the recent progress in understanding connections between gauge fields and strings that are the subject of these notes.

\subsection{The D1-D5 system}

The first success in building this kind of correspondence between black hole metrics and Dbranes was achieved by Strominger and Vafa [17]. They considered 5-dimensional supergravity obtained by compactifying 10-dimensional type IIB theory on a 5-dimensional compact manifold (for example, the 5-torus), and constructed a class of black holes carrying 2 separate $U(1)$ charges. These solutions may be viewed as generalizations of the well-known 4-dimensional charged (ReissnerNordstrom) black hole. For the Reissner-Nordstrom black hole the mass is bounded from below by a quantity proportional to the charge. In general, when the mass saturates the lower (BPS) bound for a given choice of charges, then the black hole is called extremal. The extremal StromingerVafa black hole preserves $1 / 8$ of the supersymmetries present in vacuum. Also, the black hole is constructed in such a way that, just as for the Reissner-Nordstrom solution, the area of the horizon is non-vanishing at extremality [17]. In general, an important quantity characterizing black holes is the Bekenstein-Hawking entropy which is proportional to the horizon area:

$$
S_{B H}=\frac{A_{h}}{4 G}
$$

where $G$ is the Newton constant. Strominger and Vafa calculated the Bekenstein-Hawking entropy of their extremal solution as a function of the charges and succeeded in reproducing this result with D-brane methods. To build a Dbrane system carrying the same set of charges as the black hole, they had to consider intersecting D-branes wrapped over the compact 5dimensional manifold. For example, one may consider D3-branes intersecting over a line or D1branes embedded inside D5-branes. The $1+1$ dimensional gauge theory describing such an intersection is quite complicated, but the degeneracy of the supersymmetric BPS states can nevertheless be calculated in the D-brane description valid at weak coupling. For reasons that will become clear shortly, the description in terms of black hole metrics is valid only at very strong coupling. Luckily, due to the supersymmetry, the number of states does not change as the coupling is increased. This ability to extrapolate the D-brane counting to strong coupling makes a comparison with the Bekenstein-Hawking entropy possible, and exact agreement is found in the limit of large charges [17]. In this sense the collection of D-branes provides a "microscopic" explanation of the black hole entropy.

This correspondence was quickly generalized to black holes slightly excited above the extremality $[18,19]$. Further, the Hawking radiation rates and the absorption cross-sections were calculated and successfully reproduced by D-brane models $[18,20]$. Since then this system has been receiving a great deal of attention. However, some detailed comparisons are hampered by the complexities of the dynamics of intersecting D-branes: to date there is no first principles approach to the lagrangian of the $1+1$ dimensional conformal field theory on the intersection. For this and other reasons it has turned out very fruitful to study a similar correspondence for simpler systems which involve parallel D-branes only $[21,22,23,24,25]$. We turn to this subject in the next section. 


\subsection{Coincident $\mathrm{D} p$-branes}

Our primary motivation is that, as explained above, parallel D $p$-branes realize $p+1$ dimensional $U(N)$ SYM theories, and we may learn something new about them from comparisons with Ramond-Ramond charged black $p$-brane classical solutions. These solutions in type II supergravity have been known since the early 90 's [26, 28]. The metric and dilaton backgrounds may be expressed in the following simple form:

$$
\begin{gathered}
d s^{2}=H^{-1 / 2}(r)\left[-f(r) d t^{2}+\sum_{i=1}^{p}\left(d x^{i}\right)^{2}\right]+ \\
H^{1 / 2}(r)\left[f^{-1}(r) d r^{2}+r^{2} d \Omega_{8-p}^{2}\right] \\
e^{\Phi}=H^{(3-p) / 4}(r)
\end{gathered}
$$

where

$$
H(r)=1+\frac{L^{7-p}}{r^{7-p}}, \quad f(r)=1-\frac{r_{0}^{7-p}}{r^{7-p}},
$$

and $d \Omega_{8-p}^{2}$ is the metric of a unit $8-p$ dimensional sphere. The horizon is located at $r=r_{0}$ and the extremality is achieved in the limit $r_{0} \rightarrow$ 0 . Just like the stacks of parallel D-branes, the extremal solutions are BPS saturated: they preserve 16 of the 32 supersymmetries present in the type II theory. A solution with $r_{0} \ll L$ is called near-extremal.

The correspondence between the entropies of the near-extremal $p$-brane solutions (2.2) and those of the $p+1$ dimensional SYM theories was first considered in [21, 22]. In contrast to the situation encountered for the Strominger-Vafa black hole, the Bekenstein-Hawking entropy vanishes in the extremal limit: for $r \ll L$ the longitudinal part of the metric behaves as $(r / L)^{(7-p) / 2} \sum_{i=1}^{p}\left(d x^{i}\right)^{2}$ and hence the area of the horizon vanishes. The same is obviously true on the D-brane side because the stack of D-branes is in its ground state. For $r_{0}>0$ the $p$-brane carries some excess energy $E$ above its extremal value, and the BekensteinHawking entropy is also non-vanishing. The Hawking temperature is then defined by $T^{-1}=\partial S_{B H} / \partial E$. A different, but equivalent, way of calculating the Hawking temperature is to consider a Euclidean continuation of the solutions (2.2). The Euclidean time takes values on a circle of circumference $\beta=1 / T$. As shown by Gibbons and
Hawking, $\beta$ has to be chosen in such a way that the geometry has no conical singularity at the horizon. We will use this method below to derive $T$ as a function of $r_{0}$ and $R$ in one step.

Among the solutions (2.2) $p=3$ has a special status: in the extremal limit $r_{0} \rightarrow 0$ the 3 -brane solution

$$
\begin{gathered}
d s^{2}=\left(1+\frac{L^{4}}{r^{4}}\right)^{-1 / 2}\left(-d t^{2}+d x_{1}^{2}+d x_{2}^{2}+d x_{3}^{2}\right) \\
+\left(1+\frac{L^{4}}{r^{4}}\right)^{1 / 2}\left(d r^{2}+r^{2} d \Omega_{5}^{2}\right)
\end{gathered}
$$

is perfectly non-singular [29]. One piece of evidence is that the dilaton $\Phi$ is constant for $p=3$ but blows up at $r=0$ for all other extremal solutions. Furthermore, the limiting form of the extremal metric as $r \rightarrow 0$ is

$$
d s^{2}=\frac{L^{2}}{z^{2}}\left(-d t^{2}+d \vec{x}^{2}+d z^{2}\right)+L^{2} d \Omega_{5}^{2}
$$

where $z=\frac{L^{2}}{r}$. This describes the direct product of 5-dimensional Anti-de Sitter space, $A d S_{5}$, and the 5 -dimensional sphere, $S^{5}$, with equal radii of curvature $L$ [29]. To be more precise, the above metric with $z$ ranging from 0 to $\infty$ does not cover the entire $A d S_{5}$ space, but only its Poincare wedge. This space has a horizon located at infinite $z(r=0)$. After a Euclidean continuation we obtain the entire Euclidean $A d S_{5}$ space also known as the Lobachevsky space $L_{5}$.

Since both factors of the $A d S_{5} \times S^{5}$ space (2.4) are maximally symmetric, we have

$$
R_{a b c d}=-\frac{1}{L^{2}}\left[g_{a c} g_{b d}-g_{a d} g_{b c}\right]
$$

for the $A d S_{5}$ directions, and

$$
R_{i j k l}=\frac{1}{L^{2}}\left[g_{i k} g_{j l}-g_{i l} g_{j k}\right]
$$

for the $S^{5}$ directions. This shows that near $r=$ 0 the extremal 3-brane geometry (2.3) is nonsingular and, in fact, all appropriately measured curvature components become small for large $L$. . Roughly speaking, this geometry may be viewed as a semi-infinite throat of radius $L$ which for $r \gg L$ opens up into flat $9+1$ dimensional space. Thus, for $L$ much larger than the string scale $\sqrt{\alpha^{\prime}}$, the entire 3-brane geometry has small curvatures everywhere and is appropriately described 
by the supergravity approximation to type IIB string theory.

Let us see how the requirement $L \gg \sqrt{\alpha^{\prime}}$ translates into the language of $U(N)$ SYM theory on $N$ coincident D3-branes. To this end it is convenient to equate the $\mathrm{ADM}$ tension of the extremal 3-brane classical solution to $N$ times the tension of a single D3-brane. In this fashion we find the relation $[21]$

$$
\frac{2}{\kappa^{2}} L^{4} \Omega_{5}=N \frac{\sqrt{\pi}}{\kappa}
$$

where $\Omega_{5}=\pi^{3}$ is the volume of a unit 5-sphere, and $\kappa=\sqrt{8 \pi G}$ is the 10 -dimensional gravitational constant. It follows that

$$
L^{4}=\frac{\kappa}{2 \pi^{5 / 2}} N
$$

Since $\kappa=8 \pi^{7 / 2} g_{s t} \alpha^{\prime 2},(2.8)$ gives $L^{4}=4 \pi N g_{s t} \alpha^{\prime 2}$. In turn, $g_{s t}$ determines the Yang-Mills coupling on the D3-branes through $g_{\mathrm{YM}}^{2}=2 \pi g_{s t}$. Thus, we have

$$
L^{4}=2 g_{\mathrm{YM}}^{2} N \alpha^{2},
$$

i.e. the size of the throat in string units is measured by the 't Hooft coupling! This remarkable emergence of the ' $t$ Hooft coupling from gravitational considerations is at the heart of the success of the AdS/CFT correspondence. Moreover, the requirement $L \gg \sqrt{\alpha^{\prime}}$ translates into $g_{\mathrm{YM}}^{2} N \gg 1$ : the gravitational approach is valid when the 't Hooft coupling is very strong and the traditional field theoretic methods are not applicable.

\subsection{Entropy of Near-extremal 3-branes}

Now consider the near-extremal 3-brane geometry. In the near-horizon region, $r \ll L$, we may replace $H(r)$ by $L^{4} / r^{4}$. The resulting metric [3]

$$
\begin{aligned}
d s^{2} & =\frac{r^{2}}{L^{2}}\left[-\left(1-\frac{r_{0}^{4}}{r^{4}}\right) d t^{2}+d \vec{x}^{2}\right] \\
& +\frac{L^{2}}{r^{2}}\left(1-\frac{r_{0}^{4}}{r^{4}}\right)^{-1} d r^{2}+L^{2} d \Omega_{5}^{2},
\end{aligned}
$$

is a product of $S^{5}$ with a certain limit of the Schwarzschild black hole in $A d S_{5}$ [27]. The Euclidean Schwarzschild black hole is asymptotic to $S^{1} \times S^{3}$, and the required limit is achieved as the volume of $S^{3}$ is taken to infinity. Thus, the Euclidean continuation of the metric (2.10) is asymptotic to $S^{1} \times R^{3}$. To determine the circumference of $S^{1}, \beta=1 / T$, it is convenient to set $r=r_{0}\left(1+L^{-2} \rho^{2}\right)$. For small $\rho$ the relevant $2 \mathrm{~d}$ part of the Euclidean metric is:

$$
d s^{2}=d \rho^{2}+\frac{4 r_{0}^{2}}{L^{4}} \rho^{2} d \tau^{2}, \quad \tau=i t .
$$

In order to avoid a conical singularity at the horizon, the period of the Euclidean time has to be $\beta=\pi L^{2} / r_{0}$.

The 8-dimensional "area" of the horizon can be read off from the metric (2.10). If the spatial volume of the D3-brane (i.e. the volume of the $x_{1}, x_{2}, x_{3}$ coordinates) is taken to be $V_{3}$, then we find

$$
A_{h}=\left(r_{0} / L\right)^{3} V_{3} L^{5} \Omega_{5}=\pi^{6} L^{8} T^{3} V_{3} .
$$

Using (2.8) we arrive at the Bekenstein-Hawking entropy [21]

$$
S_{B H}=\frac{2 \pi A_{h}}{\kappa^{2}}=\frac{\pi^{2}}{2} N^{2} V_{3} T^{3} .
$$

In [21] this gravitational entropy of a near-extremal 3-brane of Hawking temperature $T$ was compared with the entropy of the $\mathcal{N}=4$ supersymmetric $U(N)$ gauge theory (which lives on $N$ coincident D3-branes) heated up to the same temperature. The results turned out to be quite interesting.

The entropy of a free $U(N) \mathcal{N}=4$ supermultiplet, which consists of the gauge field, $6 \mathrm{~N}^{2}$ massless scalars and $4 N^{2}$ Weyl fermions, can be calculated using the standard statistical mechanics of a massless gas (the black body problem), and the answer is

$$
S_{0}=\frac{2 \pi^{2}}{3} N^{2} V_{3} T^{3} .
$$

It is remarkable that the 3-brane geometry captures the $T^{3}$ scaling characteristic of a conformal field theory (in a CFT this scaling is guaranteed by the extensivity of the entropy and the absence of dimensionful parameters). Also, the $N^{2}$ scaling indicates the presence of $O\left(N^{2}\right)$ unconfined degrees of freedom, which is exactly what we expect in the $\mathcal{N}=4$ supersymmetric $U(N)$ gauge theory. On the other hand, the relative factor of $3 / 4$ between $S_{B H}$ and $S_{0}$ at first appeared mysterious. In fact, this factor is not a contradiction 
but rather a prediction about the strongly coupled $\mathcal{N}=4$ SYM theory at finite temperature.

Indeed, as we argued above, the supergravity calculation of the Bekenstein-Hawking entropy, (2.13), is relevant to the $g_{\mathrm{YM}}^{2} N \rightarrow \infty$ limit of the $\mathcal{N}=4 S U(N)$ gauge theory, while the free field calculation applies to the $g_{\mathrm{YM}}^{2} N \rightarrow 0$ limit. Thus, the relative factor of $3 / 4$ is not a discrepancy: it relates two different limits of the theory. Indeed, on general field theoretic grounds we expect that in the 't Hooft large $N$ limit the entropy is given by [30]

$$
S=\frac{2 \pi^{2}}{3} N^{2} f\left(g_{\mathrm{YM}}^{2} N\right) V_{3} T^{3} .
$$

The function $f$ is certainly not constant: for example, recent calculations [31] show that its perturbative expansion is

$f\left(g_{\mathrm{YM}}^{2} N\right)=1-\frac{3}{2 \pi^{2}} g_{\mathrm{YM}}^{2} N+\frac{3+\sqrt{2}}{\pi^{3}}\left(g_{\mathrm{YM}}^{2} N\right)^{3 / 2}+\ldots$

Thus, the Bekenstein-Hawking entropy in supergravity, (2.13), is translated into the prediction that $f\left(g_{\mathrm{YM}}^{2} N \rightarrow \infty\right)=3 / 4$.

Furthermore, string theoretic corrections to the supergravity action may be used to develop a strong coupling expansion around this limiting value. The first such correction comes from the leading higher derivative term in the type IIB effective action:

$$
\begin{gathered}
I=-\frac{1}{16 \pi G} \int d^{10} x \sqrt{g}\left[R-\frac{1}{2}(\partial \phi)^{2}-\frac{1}{4 \cdot 5 !}\left(F_{5}\right)^{2}\right. \\
\left.+\ldots+\gamma e^{-\frac{3}{2} \phi} W+\ldots\right]
\end{gathered}
$$

where

$$
\gamma=\frac{1}{8} \zeta(3)\left(\alpha^{\prime}\right)^{3}
$$

and $W$ depends only on the Weyl tensor:

$$
\begin{aligned}
W= & C^{h m n k} C_{p m n q} C_{h}^{r s p} C_{r s k}^{q}+ \\
& \frac{1}{2} C^{h k m n} C_{p q m n} C_{h}^{r s p} C_{r s k}^{q} .
\end{aligned}
$$

The value of the supergravity action should be identified with the free energy of the thermal gauge theory [27]. The first correction to the free energy may be found by evaluating $O\left(\alpha^{\prime 3}\right)$ term on the leading order metric (2.10) [30]. Via the standard thermodynamics relation $S=-\frac{\partial F}{\partial T}$ this translates into the following form of the function $f$ for large ' $t$ Hooft coupling,

$$
f\left(g_{\mathrm{YM}}^{2} N\right)=\frac{3}{4}+\frac{45}{32} \zeta(3)\left(g_{\mathrm{YM}}^{2} N\right)^{-3 / 2}+\ldots .
$$

In [30] it was conjectured that $f\left(g_{\mathrm{YM}}^{2} N\right)$ is actually a monotonically decreasing function which interpolates between 1 at $g_{\mathrm{YM}}^{2} N=0$ and $3 / 4$ at $g_{\mathrm{YM}}^{2} N=\infty$. The monotonicity is consistent both with the weak coupling behavior (2.16) calculated perturbatively, and with the strong coupling behavior (2.19) found using the dual string theory.

\subsection{From absorption cross-sections to two- point correlators}

A natural step beyond the comparison of entropies is to interpret absorption cross-sections for massless particles in terms of the D-brane world volume theories [23] (for 5-d black holes the D-brane approach to absorption was initiated in $[18,20])$. For $N$ coincident D3-branes it is interesting to inquire to what extent the supergravity and the weakly coupled D-brane calculations agreed. For example, they might scale differently with $N$ or with the incident energy. Even if the scaling exponents agreed, the overall normalizations could differ by a subtle numerical factor similar to the $3 / 4$ found for the 3 -brane entropy. Surprisingly, the low-energy absorption cross-sections turn out to agree exactly [23].

To calculate the absorption cross-sections in the D-brane formalism one needs the low-energy world volume action for coincident D-branes coupled to the massless bulk fields. Luckily, these couplings may be deduced from the D-brane BornInfeld action. For example, the coupling of 3branes to the dilaton $\Phi$, the Ramond-Ramond scalar $C$, and the graviton $h_{\alpha \beta}$ is given by $[23,24]$

$$
\begin{gathered}
S_{\mathrm{int}}=\frac{\sqrt{\pi}}{\kappa} \int d^{4} x\left[\operatorname{tr}\left(\frac{1}{4} \Phi F_{\alpha \beta}^{2}-\frac{1}{4} C F_{\alpha \beta} \tilde{F}^{\alpha \beta}\right)\right. \\
\left.+\frac{1}{2} h^{\alpha \beta} T_{\alpha \beta}\right],
\end{gathered}
$$

where $T_{\alpha \beta}$ is the stress-energy tensor of the $\mathcal{N}=$ 4 SYM theory. Consider, for instance, absorption of a dilaton incident on the 3-brane at right 
angles with a low energy $\omega$. Since the dilaton couples to $\frac{1}{4} \operatorname{tr} F_{\alpha \beta}^{2}$ it can be converted into a pair of back-to-back gluons on the world volume. The leading order calculation of the cross-section for weak coupling gives [23]

$$
\sigma=\frac{\kappa^{2} \omega^{3} N^{2}}{32 \pi}
$$

The factor $N^{2}$ comes from the degeneracy of the final states which is the number of different gluon species.

This result was compared with the absorption cross-section by the extremal 3-brane geometry (2.3). As discussed above, the geometry is a non-singular semi-infinite throat which opens up at large $r$ into flat 10-dimensional space. Waves incident from the $r \gg L$ region partly reflect back and partly penetrate into the the throat region $r \ll L$. The relevant s-wave radial equation turns out to be [23]

$$
\left[\frac{d^{2}}{d \rho^{2}}-\frac{15}{4 \rho^{2}}+1+\frac{(\omega L)^{4}}{\rho^{4}}\right] \psi(\rho)=0,
$$

where $\rho=\omega r$. For a low energy $\omega \ll 1 / L$ we find a high barrier separating the two asymptotic regions. The low-energy behavior of the tunneling probability may be calculated by the so-called matching method, and the resulting absorption cross-section is [23]

$$
\sigma_{S U G R A}=\frac{\pi^{4}}{8} \omega^{3} L^{8}
$$

Substituting (2.8) we find that the supergravity absorption cross-section agrees exactly with the D-brane one, without any relative factor like $3 / 4$.

This surprising result needs an explanation. The most important question is: what is the range of validity of the two calculations? The supergravity approach may be trusted only if the length scale of the 3 -brane solution is much larger than the string scale $\sqrt{\alpha^{\prime}}$. As we have shown, this translates into $N g_{s t} \gg 1$. Of course, the incident energy also has to be small compared to $1 / \sqrt{\alpha^{\prime}}$. Thus, the supergravity calculation should be valid in the "double-scaling limit" [23]

$$
\frac{L^{4}}{\alpha^{2}}=4 \pi g_{s t} N \rightarrow \infty, \quad \omega^{2} \alpha^{\prime} \rightarrow 0 .
$$

If the description of the black 3-brane by a stack of many coincident D3-branes is correct, then it must agree with the supergravity results in this limit, which corresponds to infinite " $t$ Hooft coupling in the $\mathcal{N}=4 U(N)$ SYM theory. Since we also want to send $g_{s t} \rightarrow 0$ in order to suppress the string loop corrections, we necessarily have to take the large $N$ limit.

Although we have sharpened the region of applicability of the supergravity calculation (2.23), we have not yet explained why it agrees with the leading order perturbative result (2.21) on the D3-brane world volume. After including the higher-order SYM corrections, the general structure of the absorption cross-section in the large $N$ limit is expected to be [25]

$$
\sigma=\frac{\kappa^{2} \omega^{3} N^{2}}{32 \pi} a\left(g_{\mathrm{YM}}^{2} N\right)
$$

where

$$
a\left(g_{\mathrm{YM}}^{2} N\right)=1+b_{1} g_{\mathrm{YM}}^{2} N+b_{2}\left(g_{\mathrm{YM}}^{2} N\right)^{2}+\ldots
$$

For agreement with supergravity, the strong 't Hooft coupling limit of $a\left(g_{\mathrm{YM}}^{2} N\right)$ should be equal to 1 [25]. In fact, a stronger result is true: all perturbative corrections vanish and $a=1$ independent of the coupling. This was first shown explicitly in [25] for the graviton absorption. The absorption cross-section for a graviton polarized along the brane, say $h_{x y}$, is related to the discontinuity accross the real axis (i.e. the absorptive part) of the two-point function $\left\langle T_{x y}(p) T_{x y}(-p)\right\rangle$ in the SYM theory. In turn, this is determined by a conformal "central charge" which satisfies a non-renormalization theorem: it is completely independent of the ' $t$ Hooft coupling.

In general, the two-point function of a gauge invariant operator in the strongly coupled SYM theory may be read off from the absorption crosssection for the supergravity field which couples to this operator in the world volume action [25,32]. Consider, for instance, scalar operators. For a canonically normalized bulk scalar field coupling to the brane through an interaction

$$
S_{\mathrm{int}}=\int d^{4} x \phi(x, 0) \mathcal{O}(x)
$$

$(\phi(x, 0)$ denotes the value of the field at the transverse coordinates where the D3-branes are lo- 
cated) the precise relation is given by

$$
\sigma=\left.\frac{1}{2 i \omega} \operatorname{Disc} \Pi(p)\right|_{-p^{2}=\omega^{2}-i \epsilon} ^{-p^{2}=\omega^{2}+i \epsilon}
$$

Here $\omega$ is the energy of the particle, and

$$
\Pi(p)=\int d^{4} x e^{i p \cdot x}\langle\mathcal{O}(x) \mathcal{O}(0)\rangle
$$

which depends only on $s=-p^{2}$. To evaluate (2.27) we extend $\Pi$ to complex values of $s$ and compute the discontinuity of $\Pi$ across the real axis at $s=\omega^{2}$.

Some examples of the field operator correspondence may be read off from (2.20). Thus, we learn that the dilaton absorption cross-section measures the normalized 2-point function $\left\langle O_{\Phi}(p) O_{\Phi}(-p)\right\rangle$ where $O_{\Phi}$ is the operator that couples to the dilaton:

$$
O_{\Phi}=\frac{1}{4} \operatorname{tr}\left(F^{2}+\ldots\right)
$$

(we have not written out the scalar and fermion terms explicitly). Similarly, the Ramond-Ramond scalar absorption cross-section measures $\left\langle O_{C}(p) O_{C}(-p)\right.$ where

$$
O_{C}=\frac{1}{4} \operatorname{tr} F_{\alpha \beta} \tilde{F}^{\alpha \beta}+\ldots
$$

The agreement of these two-point functions with the weak-coupling calculations performed in [23, $24]$ is explained by non-renormalization theorems related by supersymmetry to the non-renormalization of the central charge discussed in [25]. Thus, the proposition that the $g_{\mathrm{YM}}^{2} N \rightarrow \infty$ limit of the large $N \mathcal{N}=4$ SYM theory can be extracted from the 3-brane of type IIB supergravity has passed its first consistency checks.

It is of further interest to perform similar comparisons in cases where the relevant non-renormalization theorems have not yet been proven. Consider, for instance, absorption of the dilaton in the $l$-th partial wave. Now the angular laplacian on $S^{5}$ has the eigenvalue $l(l+4)$ and the effective radial equation becomes

$$
\left[\frac{d^{2}}{d \rho^{2}}-\frac{l(l+4)+15 / 4}{\rho^{2}}+1+\frac{(\omega L)^{4}}{\rho^{4}}\right] \psi(\rho)=0,
$$

The thickness of the barrier through which the particle has to tunnel increases with $l$, and we expect the cross-section to become increasingly suppressed at low energies. Indeed, a detailed matching calculation $[24,32]$ gives

$$
\sigma_{S U G R A}^{l}=\frac{\pi^{4}}{24} \frac{(l+3)(l+1)}{[(l+1) !]^{4}}\left(\frac{\omega L}{2}\right)^{4 l} \omega^{3} L^{8} .
$$

Replacing $L^{4}$ through (2.8) this can be rewritten as

$$
\sigma^{l}=\frac{N^{l+2} \kappa^{l+2} \omega^{4 l+3}(l+3)}{3 \cdot 2^{5 l+5} \pi^{5 l / 2+1} l ![(l+1) !]^{3}} .
$$

What are the operators whose 2-point functions are related to these cross-sections? For a single D3-brane one may expand the dilaton coupling in a Taylor series in the transverse coordinates to obtain the following bosonic term [23]:

$$
\frac{1}{4 l !} F_{\alpha \beta} F^{\alpha \beta} X^{\left(i_{1}\right.} \ldots X^{\left.i_{l}\right)}
$$

where the parenthesis pick out a transverse traceless polynomial, which is an irreducible representation of $S O(6)$. The correct non-abelian generalization of this term is [32]

$$
\frac{1}{4 l !} \operatorname{STr}\left[F_{\alpha \beta} F^{\alpha \beta} X^{\left(i_{1}\right.} \ldots X^{\left.i_{l}\right)}\right],
$$

where STr denotes a symmetrized trace [33]: in this particular case we have to average over all positions of the F's modulo cyclic permutations. A detailed calculation in [32] reveals that the 2point function of this operator calculated at weak coupling accounts for $\frac{6}{(l+2)(l+3)}$ of the semiclassical absorption cross-section (2.33) in the sense of the relation (2.27). Luckily, (2.35) is not the complete world volume coupling to the dilaton in the $l$-th partial wave. $\mathcal{N}=4$ supersymmetry guarantees that there are additional terms quadratic and quartic in the fermion fields. When all these terms are taken into account there is $e x$ act agreement between the weak and strong coupling calculations of the 2-point functions. This strongly suggests that the complete $l$-th partial wave operators are protected by supersymmetric non-renormalization theorems. Proving them is an interesting challenge (for recent progress, see $[34])$.

\section{The AdS/CFT Correspondence}

The circle of ideas reviewed in the previous sections received an important development by Maldacena [3] who also connected it for the first time 
with the QCD string idea. Maldacena made a simple and powerful observation that the "universal" region of the 3 -brane geometry, which should be directly identified with the $\mathcal{N}=4$ SYM theory, is the throat, i.e. the region $r \ll L .^{1}$ The limiting form of the 3 -brane metric (2.3) is (2.4) which describes the space $A d S_{5} \times S^{5}$ with equal radii of curvature $L$. One also finds that the self-dual 5-form R-R field strength has $N$ units of flux through this space (the field strength term in the Einstein equation effectively gives a positive cosmological constant on $S^{5}$ and a negative one on $A d S_{5}$ ). Thus, Maldacena conjectured that type IIB string theory on $A d S_{5} \times S^{5}$ should be somehow dual to the $\mathcal{N}=4$ supersymmetric $S U(N)$ gauge theory.

By the same token, identifying, as we did in the preceding section, the 2-brane and 5-brane classical solutions of 11-dimensional supergravity with stacks of M2 and M5 branes respectively leads to similar dualities in the M-theory context. In particular, a large $N$ 6-dimensional $(2,0)$ theory is conjectured to be dual to the $A d S_{7} \times S^{4}$ background, and a large $N$ maximally supersymmetric 3-dimensional gauge theory is conjectured to be dual to the $A d S_{4} \times S^{7}$ background. In the following we will discuss only the D3-brane case, but generalization to the M-branes is straightforward.

Maldacena's argument was based on the fact that the low-energy $\left(\alpha^{\prime} \rightarrow 0\right)$ limit may be taken directly in the 3-brane geometry and is equivalent to the throat $(r \rightarrow 0)$ limit. Another way to motivate the identification of the gauge theory with the throat is to think about the absorption of massless particles considered in the previous section. In the D-brane description, a particle incident from the asymptotic infinity is converted into an excitation of the stack of D-branes, i.e. into an excitation of the gauge theory on the world volume. In the supergravity description, a particle incident from the asymptotic (large $r$ ) region tunnels into the $r \ll L$ region and produces an excitation of the throat. The fact that the two different descriptions of the absorption process give identical cross-sections supports the identification of excitations of $A d S_{5} \times S^{5}$ with

\footnotetext{
${ }^{1}$ Related ideas were also pursued in [35].
}

the excited states of the $\mathcal{N}=4$ SYM theory.

Another strong piece of support for this identification comes from symmetry considerations [3]. The isometry group of $A d S_{5}$ is $S O(2,4)$, and this is also the conformal group in $3+1$ dimensions. In addition we have the isometries of $S^{5}$ which form $S U(4) \sim S O(6)$. This group is identical to the R-symmetry of the $\mathcal{N}=4 \mathrm{SYM}$ theory. After including the fermionic generators required by supersymmetry, the full isometry supergroup of the $A d S_{5} \times S^{5}$ background is $S U(2,2 \mid 4)$, which is identical to the $\mathcal{N}=4$ superconformal symmetry. We will see that in theories with reduced supersymmetry the compact $S^{5}$ factor becomes replaced by other compact spaces $X_{5}$, but $A d S_{5}$ is the "universal" factor present in the dual description of any large $N$ CFT and realizing the $S O(2,4)$ conformal symmetry. One may think of these backgrounds as type IIB theory compactified on $X_{5}$ down to 5 dimensions. Such KaluzaKlein compactifications of type IIB supergravity were extensively studied in the mid-eighties $[36,37,38]$, and special attention was devoted to the $A d S_{5} \times S^{5}$ solution because it is a maximally supersymmetric background [39, 40]. It is remarkable that these early works on compactification of type IIB theory were actually solving large $N$ gauge theories without knowing it.

As Maldacena has emphasized, it is also important to go beyond the supergravity limit and think of the $A d S_{5} \times X_{5}$ space as a background of string theory [3]. Indeed, type IIB strings are dual to the electric flux lines in the gauge theory, and this provides a natural set-up for calculating correlation functions of the Wilson loops [41]. Furthermore, if $N$ is sent to infinity while $g_{\mathrm{YM}}^{2} N$ is held fixed and finite, then there are finite string scale corrections to the supergravity limit $[3,4,5]$ which proceed in powers of $\frac{\alpha^{\prime}}{L^{2}}=\left(2 g_{\mathrm{YM}}^{2} N\right)^{-1 / 2}$. If we wish to study finite $N$, then there are also string loop corrections in powers of $\frac{\kappa^{2}}{L^{8}} \sim N^{-2}$. As expected, taking $N$ to infinity enables us to take the classical limit of the string theory on $A d S_{5} \times X_{5}$. However, in order to understand the large $N$ gauge theory with finite 't Hooft coupling, we should think of the $A d S_{5} \times X_{5}$ as the target space of a 2-dimensional sigma model describing the classical string physics [4]. The fact 
that after the compactification on $X_{5}$ the string theory is 5-dimensional supports Polyakov's idea [8]. In $A d S_{5}$ the fifth dimension is related to the radial coordinate and, after a change of variables $z=L e^{-\varphi / L}$, the sigma model action turns into a special case of the general ansatz proposed in $[8]$,

$$
S=\frac{1}{2} \int d^{2} \sigma\left[\left(\partial_{\alpha} \varphi\right)^{2}+a^{2}(\varphi)\left(\partial_{\alpha} X^{i}\right)^{2}+\ldots\right],
$$

with $a(\varphi)=e^{\varphi / L}$. It is clear, however, that the string sigma models dual to the gauge theories are of rather peculiar nature. The new feature revealed by the D-brane approach, which is also a major stumbling block, is the presence of the Ramond-Ramond background fields. Little is known to date about such 2-dimensional field theories and, in spite of recent new insights [42], an explicit solution is not yet available.

\subsection{Correlation functions and the bulk-bound correspondence}

Maldacena's work provided a crucial insight that the $A d S_{5} \times S^{5}$ throat is the part of the 3 -brane geometry that is most directly related to the $\mathcal{N}=4$ SYM theory. It is important to go further, however, and explain precisely in what sense the two should be identified and how physical information can be extracted from this duality. Major strides towards answering these questions were made in two subsequent papers $[4,5]$ where essentially identical methods for calculating correlation functions of various operators in the gauge theory were proposed. As we mentioned in section 2.2, even prior to [3] some information about the field/operator correspondence and about the two-point functions had been extracted from the absorption cross-sections. The reasoning of [4] was a natural extension of these ideas.

One may motivate the general method as follows. When a wave is absorbed, it tunnels from the asymptotic infinity into the throat region, and then continues to propagate toward smaller $r$. Let us separate the 3-brane geometry into two regions: $r \gtrsim L$ and $r \lesssim L$. For $r \lesssim L$ the metric is approximately that of $A d S_{5} \times S^{5}$, while for $r \gtrsim L$ it becomes very different and eventually approaches the flat metric. Signals coming in from large $r$ may be thought of as disturbing the "boundary" of $A d S_{5}$ at $r \sim L$, and then propagating into the bulk. This suggests that, if we discard the $r \gtrsim L$ part of the 3-brane metric, then the gauge theory correlation functions are related to the response of the string theory to boundary conditions at $r \sim L$. Guided by this idea, [4] proposed to identify the generating functional of connected correlation functions in the gauge theory with the extremum of the classical string theory action $I$ subject to the boundary conditions that $\phi\left(x^{\lambda}, z\right)=\phi_{0}\left(x^{\lambda}\right)$ at $z=L$ (at $z=\infty$ all fluctuations are required to vanish): ${ }^{2}$

$$
W\left[\phi_{0}\left(x^{\lambda}\right)\right]=I_{\phi_{0}\left(x^{\lambda}\right)} .
$$

$W$ generates the connected Green's functions of the gauge theory operator that corresponds to the field $\phi$ in the sense explained in section 2.2, while $I_{\phi_{0}\left(x^{\lambda}\right)}$ is the extremum of the classical string action subject to the boundary conditions.

ardyn essentially identical prescription was also proposed in [5] with a somewhat different motivation. If we are interested in the correlation functions at infinite 't Hooft coupling, then the problem of extremizing the classical string action reduces to solving the equations of motion in type IIB supergravity whose form is known explicitly [39].

Our reasoning suggests that from the point of view of the metric (2.4) the boundary conditions are imposed not at $z=0$ (which would be a true boundary of $A d S_{5}$ ) but at some finite value $z=z_{\text {cutof } f}$. It does not matter which value it is since the metric (2.4) is unchanged by an overall rescaling of the coordinates $\left(z, x^{\lambda}\right)$; thus, such a rescaling can take $z=L$ into $z=z_{\text {cutoff }}$ for any $z_{\text {cutoff }}$. The physical meaning of this cut-off is that it acts as a UV regulator in the gauge theory [ 4,43$]$. Indeed, the radial coordinate of $A d S_{5}$ is to be thought of as the effective energy scale of the gauge theory [3], and decreasing $z$ corresponds to increasing the energy. A safe method for performing calculations of correlation functions, therefore, is to keep the cut-off on the $z$ coordinate at intermediate stages and remove it

\footnotetext{
${ }^{2}$ As usual, in calculating correlation functions in a CFT it is convenient to carry out the Euclidean continuation. On the string theory side we then have to use the Euclidean version of $A d S_{5}$.
} 
only at the end $[4,44]$. This way the calculations are not manifestly AdS-invariant, however. Usually there is another way to regularize the action which is manifestly AdS invariant. Luckily, when all subtleties are taken into account, these two ways of performing calculations do agree [45].

\subsection{Two-point functions}

Below we present a brief discussion of two-point functions of scalar operators. The corresponding field in $A d S_{d+1}$ is a scalar field of mass $m$ with the action

$$
\begin{gathered}
\frac{1}{2} \int d^{d+1} x \sqrt{g}\left[g^{\mu \nu} \partial_{\mu} \phi \partial_{\nu} \phi+m^{2} \phi^{2}\right]= \\
\frac{1}{2} \int d^{d} x d z z^{-d+1}\left[\left(\partial_{z} \phi\right)^{2}+\left(\partial_{i} \phi\right)^{2}+\frac{m^{2}}{z^{2}} \phi^{2}\right],
\end{gathered}
$$

where we have set $L=1$. In calculating correlation functions of vertex operators from the AdS/CFT correspondence, the first problem is to reconstruct an on-shell field in $A d S_{d+1}$ from its boundary behavior. The small $z$ behavior of the classical solution is

$\phi(z, \vec{x}) \rightarrow z^{d-\Delta}\left[\phi_{0}(\vec{x})+O\left(z^{2}\right)\right]+z^{\Delta}\left[A(\vec{x})+O\left(z^{2}\right)\right]$,

where $\Delta$ is one of the roots of

$$
\Delta(\Delta-d)=m^{2}
$$

$\phi_{0}(\vec{x})$ is regarded as a "source" function and $A(\vec{x})$ describes a physical fluctuation.

It is possible to regularize the Euclidean action [45] to obtain the following value as a functional of the source,

$$
\begin{gathered}
I\left(\phi_{0}\right)=-(\Delta-(d / 2)) \pi^{-d / 2} \frac{\Gamma(\Delta)}{\Gamma(\Delta-(d / 2))} \\
\int d^{d} \vec{x} \int d^{d} \vec{x}^{\prime} \frac{\phi_{0}(\vec{x}) \phi_{0}\left(\vec{x}^{\prime}\right)}{\left|\vec{x}-\vec{x}^{\prime}\right|^{2 \Delta}} .
\end{gathered}
$$

Varying twice with respect to $\phi_{0}$ we find that the two-point function of the corresponding operator is

$$
\left\langle\mathcal{O}(\vec{x}) \mathcal{O}\left(\vec{x}^{\prime}\right)\right\rangle=\frac{(2 \Delta-d) \Gamma(\Delta)}{\pi^{d / 2} \Gamma(\Delta-(d / 2))} \frac{1}{\left|\vec{x}-\vec{x}^{\prime}\right|^{2 \Delta}} .
$$

Precisely the same normalization of the two-point function follows from a different regularization where $z_{\text {cutoff }}$ is kept at intermediate stages [4, 44].

We note that $\Delta$ is the dimension of the operator. Which of the two roots of (3.5) should we choose? Superficially it seems that we should always choose the bigger root,

$$
\Delta_{+}=\frac{d}{2}+\sqrt{\frac{d^{2}}{4}+m^{2}}
$$

because then the $\phi_{0}$ term in (3.4) dominates over the $A$ term. While for positive $m^{2} \Delta_{+}$is certainly the right choice (here the other root $\Delta_{-}$is negative), it turns out that for

$$
-\frac{d^{2}}{4}<m^{2}<-\frac{d^{2}}{4}+1
$$

both roots of (3.5) may be chosen. Thus, there are two possible CFT's corresponding to the same classical AdS action [45]: in one of them the corresponding operator has dimension $\Delta_{+}$while in the other - dimension $\Delta_{-}$. (The fact that there are two admissible boundary conditions in $A d S_{d+1}$ for a scalar field with $m^{2}$ in the range (3.9) has been known since the old work of Breitenlohner and Freedman [46].) This conclusion resolves the following puzzle. $\Delta_{+}$is bounded from below by $d / 2$ but there is no corresponding bound in $d$-dimensional CFT (in fact, as we will see, there are examples of field theories with operators that violate this bound). However, in the range (3.9) $\Delta_{-}$is bounded from below by $(d-$ $2) / 2$, and this is precisely the unitarity bound on dimensions of scalar operators in $d$-dimensional field theory! Thus, the ability to have dimension $\Delta_{-}$is crucial for consistency of the AdS/CFT duality.

A question remains, however, as to what is the correct definition of correlation functions in the theory with dimension $\Delta_{-}$. The answer to this question is related to the physical interpretation of the function $A(\vec{x})$ entering the boundary behavior of the field (3.4). As suggested in [47] this function is related to the expectation value of the operator $\mathcal{O}$. The precise relation, which holds even after interactions are taken into account, is [45]

$$
A(\vec{x})=\frac{1}{2 \Delta-d}\langle\mathcal{O}(\vec{x})\rangle
$$

Thus, from the point of view of the $d$-dimensional CFT, $(2 \Delta-d) A(\vec{x})$ is the variable conjugate to 
$\phi_{0}(\vec{x})$. In order to interchange $\Delta$ with $d-\Delta$, it is clear from (3.4) that we have to interchange $\phi_{0}$ and $(2 \Delta-d) A$. This is a canonical transformation which for tree-level correlators reduces to a Legendre transform. Thus, the generating functional of correlators in the $\Delta_{-}$theory may be obtained by Legendre transforming the generating functional of correlators in the $\Delta_{+}$theory. This gives a simple and explicit prescription for defining correlation functions of operators with dimension $\Delta_{-}$. For the 2-point function, for example, we find that the formula (3.7) is correct for both definitions of the theory, i.e. it makes sense for all dimensions above the untarity bound,

$$
\Delta>\frac{d}{2}-1
$$

Indeed, note that for such dimensions the twopoint function (3.7) is positive, but as soon as $\Delta$ crosses the unitarity bound, (3.7) becomes negative signaling a non-unitarity of the theory. Thus, appropriate treatment of fields in $A d S_{d+1}$ gives information on 2-point functions completely consistent with expectations from $\mathrm{CFT}_{d}$. The fact that the Legendre transform prescription of [45] works properly for higher-point correlation functions was recently demonstrated in [48].

Whether string theory on $A d S_{5} \times X_{5}$ contains fields with mass-squared in the range (3.9) depends on $X_{5}$. The example discussed in section 4, $X_{5}=T^{1,1}$, turns out to contain such fields, and the possibility of having dimension $\Delta_{-}$is crucial for the consistency of the AdS/CFT duality. However, for $X_{5}=S^{5}$ which is dual to the $\mathcal{N}=4$ large $N$ SYM theory, there are no such fields and all scalar dimensions are given by (3.8) (to reinstate $L$ we simply replace $m$ by $m L$ ).

The operators in the $\mathcal{N}=4$ large $N$ SYM theory naturally break up into two classes: those that correspond to the Kaluza-Klein states of supergravity and those that correspond to massive string states. Since the radius of the $S^{5}$ is $L$, the masses of the Kaluza-Klein states are proportional to $1 / L$. Thus, the dimensions of the corresponding operators are independent of $L$ and therefore independent of $g_{\mathrm{YM}}^{2} N$. On the gauge theory side this is explained by the fact that the supersymmetry protects the dimensions of certain operators from being renormalized: they are completely determined by the representation under the superconformal symmetry $[49,50]$. All families of the Kaluza-Klein states, which correspond to such BPS protected operators, were classified long ago [40]. Correlation functions of such operators in the strong 't Hooft coupling limit may be obtained from the dependence of the supergravity action on the boundary values of corresponding Kaluza-Klein fields, as in (3.2). A variety of explicit calculations have been performed for 2-, 3- and even 4-point functions. The 4-point functions are particularly interesting because their dependence on operator positions is not determined by the conformal invariance. For state-of-the-art results on them, see [51, 52].

On the other hand, the masses of string excitations are $m^{2}=\frac{4 n}{\alpha^{\prime}}$ where $n$ is an integer. For the corresponding operators the formula (3.8) predicts that the dimensions do depend on the 't Hooft coupling and, in fact, blow up for large $g_{\mathrm{YM}}^{2} N$ as $2\left(n g_{\mathrm{YM}} \sqrt{2 N}\right)^{1 / 2}$. This is a highly non-trivial prediction of the AdS/CFT duality which has not yet been verified on the gauge theory side.

\section{Conformal field theories and Ein- stein manifolds}

As we mentioned above, the duality between type IIB strings on $A d S_{5} \times S^{5}$ and the $\mathcal{N}=4 \mathrm{SYM}$ is naturally generalized to dualities between backgrounds of the form $A d S_{5} \times X_{5}$ and other conformal gauge theories. The 5-dimensional compact space $X_{5}$ is required to be a postively curved Einstein manifold, i.e. one for which $R_{\mu \nu}=\Lambda g_{\mu \nu}$ with $\Lambda>0$. The number of supersymmetries in the dual gauge theory is determined by the number of Killing spinors on $X_{5}$.

The simplest examples of $X_{5}$ are the orbifolds $S^{5} / \Gamma$ where $\Gamma$ is a discrete subgroup of $S O(6)[10,11]$. In these cases $X_{5}$ has the local geometry of a 5-sphere. The dual gauge theory is the IR limit of the world volume theory on a stack of $N$ D3-branes placed at the orbifold singularity of $R^{6} / \Gamma$. Such theories typically involve product gauge groups $S U(N)^{k}$ coupled to matter in bifundamental representations [53]. 
Constructions of the dual gauge theories for Einstein manifolds $X_{5}$ which are not locally equivalent to $S^{5}$ are also possible. The simplest example is the Romans compactification on $X_{5}=$ $T^{1,1}=(S U(2) \times S U(2)) / U(1)[37,12]$. It turns out that the dual gauge theory is the conformal limit of the world volume theory on a stack of $N$ D3-branes placed at the singularity of a certain Calabi-Yau manifold known as the conifold [12]. Let us explain this connection in more detail.

The conifold may be described by the following equation in four complex variables,

$$
\sum_{a=1}^{4} z_{a}^{2}=0
$$

Since this equation is symmetric under an overall rescaling of the coordinates, this space is a cone. Remarkably, the base of this cone is precisely the space $T^{1,1}[54,12]$. A simple argument in favor of this is based on the symmetries. In order to find the base we intersect (4.1) with $\sum_{a=1}^{4}\left|z_{a}\right|^{2}=1$. The resulting space has the $S O(4)$ symmetry which rotates the $z$ 's, and also the $U(1)$ R-symmetry under $z_{a} \rightarrow e^{i \theta} z_{a}$. Since $S O(4) \sim S U(2) \times S U(2)$ these symmetries coincide with those of $T^{1,1}$. In fact, the metric on the conifold may be cast in the form [54]

$$
d s_{6}^{2}=d r^{2}+r^{2} d s_{5}^{2},
$$

where $d s_{5}^{2}$ is the metric on $T^{1,1}$. Now placing $N$ D3-branes at the apex of the cone we find the metric

$$
\begin{gathered}
d s^{2}=\left(1+\frac{L^{4}}{r^{4}}\right)^{-1 / 2}\left(-d t^{2}+d x_{1}^{2}+d x_{2}^{2}+d x_{3}^{2}\right) \\
+\left(1+\frac{L^{4}}{r^{4}}\right)^{1 / 2}\left(d r^{2}+r^{2} d s_{5}^{2}\right)
\end{gathered}
$$

whose near-horizon limit is $A d S_{5} \times T^{1,1}$ (once again, $\left.L^{4} \sim g_{s} N\right)$. Thus, type IIB string theory on this space should be dual to the infrared limit of the field theory on $N$ D3-branes placed at the singularity of the conifold. Since Calabi-Yau spaces preserve $1 / 4$ of the original supersymmetries we find that this should be an $\mathcal{N}=1$ superconformal field theory. This field theory was first constructed in [12]: it is $S U(N) \times S U(N)$ gauge theory coupled to two chiral superfields,
$A_{i}$, in the $(\mathbf{N}, \overline{\mathbf{N}})$ representation and two chiral superfields, $B_{j}$, in the $(\overline{\mathbf{N}}, \mathbf{N})$ representation [12]. The $A$ 's transform as a doublet under one of the global $S U(2)$ 's while the $B$ 's transform as a doublet under the other $S U(2)$.

Cancellation of the anomaly in the $U(1) \mathrm{R}$ symmetry requires that the $A$ 's and the $B$ 's each have R-charge $1 / 2$. For consistency of the duality it is necessary that we add an exactly marginal superpotential which preserves the $S U(2) \times S U(2) \times$ $U(1)_{R}$ symmetry of the theory (this superpotential produces a critical line related to the radius of $\left.A d S_{5} \times T^{1,1}\right)$. Since a marginal superpotential has R-charge equal to 2 it must be quartic, and the symmetries fix it uniquely up to overall normalization:

$$
W=\epsilon^{i j} \epsilon^{k l} \operatorname{tr} A_{i} B_{k} A_{j} B_{l} .
$$

There is a number of convincing checks of the duality between this field theory and type IIB strings on $A d S_{5} \times T^{1,1}$. We will point out one subtle check which is related to the discussion of the operator dimensions in section 3.2. The simplest chiral operators were constructed in [12]:

$$
\operatorname{tr}\left(A_{i_{1}} B_{j_{1}} \ldots A_{i_{k}} B_{j_{k}}\right) .
$$

Since the F-term constraints in the gauge theory require that the $i$ and the $j$ indices are separately symmetrized, we find that their $S U(2) \times S U(2)$ quantum numbers are $(k / 2, k / 2)$. The R-charge is $k$ which determines the operator dimensions to be $\Delta=3 k / 2$. This spectrum of quantum numbers and dimensions indeed follows via the AdS/CFT correspondence from the spectrum of type IIB string theory on $A d S_{5} \times T^{1,1}[12,55,56]$. Let us emphasize one interesting subtlety: the dimension corresponding to the $k=1$ operators, $3 / 2$, is below $d / 2=2$. Thus, it must correspond to the smaller root of (3.5), $\Delta_{-}$. Analysis of the spectrum of type IIB theory on $A d S_{5} \times T^{1,1}$ $[55,56]$ reveals the presence of a scalar with $m^{2}=$ $-15 / 4 L^{2}$ which, when substituted into the formula for $\Delta_{-}$, indeed gives $3 / 2$.

In fact, the supermultiplet containing this scalar includes another scalar with $m^{2}=-15 / 4 L^{2}$ and a massless fermion [56]. One of these scalar fields corresponds to the lower component of the superfield $\operatorname{tr}\left(A_{i} B_{j}\right)$, which has dimension $3 / 2$, 
while the other corresponds to the upper component which has dimension $5 / 2$. Thus, the supersymmetry requires that we pick dimension $\Delta_{+}$ for one of the conformally coupled scalars, and $\Delta_{-}$for the other. This is but one of many examples of how supersymmetry helps in constraining and testing the gauge theory/gravity correspondence.

\section{Acknowledgements}

I am grateful to S. Gubser, A. Peet, A. Polyakov, A. Tseytlin and E. Witten, my collaborators on parts of the material reviewed in these notes. This work was supported in part by the NSF grant PHY-9802484 and by the James S. McDonnell Foundation Grant No. 91-48.

\section{References}

[1] Y. Nambu, "Quark model and the factorization of the Veneziano amplitude," in Symmetries and Quark Models, ed. R. Chand, Gordon and Breach (1970); H. B. Nielsen, "An almost physical interpretation of the integrand of the n-point Veneziano amplitude," submitted to the 15th International Conference on High Energy Physics, Kiev (1970); L. Susskind, "Dualsymmetric theory of hadrons," Nuovo Cim. 69A (1970) 457.

[2] J. Scherk and J. Schwarz, "Dual models for nonhadrons," Nucl. Phys. B81 (1974) 118.

[3] J. Maldacena, "The Large $\mathrm{N}$ limit of superconformal field theories and supergravity," Adv. Theor. Math. Phys. 2 (1998) 231, hep-th/9711200.

[4] S.S. Gubser, I.R. Klebanov, and A.M. Polyakov, "Gauge theory correlators from noncritical string theory," Phys. Lett. B428 (1998) 105, hep-th/9802109.

[5] E. Witten, "Anti-de Sitter space and holography," Adv. Theor. Math. Phys. 2 (1998) 253, hep-th/9802150.

[6] G. 't Hooft, "A planar diagram theory for strong interactions,"

Nucl. Phys. B72 (1974) 461.
[7] See, for example, A.M. Polyakov, "Gauge Fields and Strings," Harwood Academic Publishers (1987).

[8] A.M. Polyakov, "String theory and quark confinement," Nucl. Phys. B (Proc. Suppl.) 68 (1998) 1, hep-th/9711002.

[9] A.M. Polyakov, "Quantum geometry of bosonic strings," Phys. Lett. B103 (1981) 207.

[10] S. Kachru and E. Silverstein, "4d conformal field theories and strings on orbifolds," hep-th/9802183.

[11] A. Lawrence, N. Nekrasov and C. Vafa, "On conformal field theories in four dimensions," hep-th/9803015.

[12] I.R. Klebanov and E. Witten, "Superconformal field theory on threebranes at a CalabiYau singularity," Nucl. Phys. B536 (1998) 199, hep-th/9807080.

[13] For a review, see J. Polchinski, "TASI lectures on D-branes," hep-th/9611050.

[14] J. Polchinski, "Dirichlet branes and RamondRamond charges," Phys. Rev. Lett. 75 (1995) 4724, hep-th/9510017.

[15] C.M. Hull and P.K. Townsend, "Unity of superstring dualities," Nucl. Phys. B438 (1995) 109; P.K. Townsend, "The eleven-dimensional supermembrane revisited," Phys. Lett. B350 (1995) 184; E. Witten, "String theory dynamics in various dimensions," Nucl. Phys. B443 (1995) 85.

[16] E. Witten, "Bound states of strings and p-branes," Nucl. Phys. B460 (1996) 335, hep-th/9510135.

[17] A. Strominger and C. Vafa, "Microscopic origin of the Bekenstein-Hawking entropy," Phys. Lett. B379 (1996) 99 , hep-th/9601029.

[18] C.G. Callan and J.M. Maldacena, "D-brane approach to black hole quantum mechanics," Nucl. Phys. B472 (1996) 591, hep-th/9602043.

[19] G. Horowitz and A. Strominger, "Counting states of near-extremal black holes," Phys. Rev. Lett. 77 (1996) 2368, hep-th/9602051. 
[20] A. Dhar, G. Mandal and S. Wadia, "Absorption vs. decay of black holes in string theory and T symmetry," Phys. Lett. B388 (1996) 51; S. Das and S. Mathur, "Comparing decay rates for black holes and D-branes," Nucl. Phys. B478 (1996) 561.

[21] S.S. Gubser, I.R. Klebanov, and A.W. Peet, "Entropy and temperature of black 3-branes," Phys. Rev. D54 (1996) 3915, hep-th/9602135; A. Strominger, unpublished.

[22] I.R. Klebanov and A.A. Tseytlin, "Entropy of near-extremal black p-branes," Nucl. Phys. B475 (1996) 164, hep-th/9604089.

[23] I.R. Klebanov, "World volume approach to absorption by nondilatonic branes," Nucl. Phys. B496 (1997) 231, hep-th/9702076.

[24] S.S. Gubser, I.R. Klebanov, and A.A. Tseytlin, "String theory and classical absorption by three-branes," Nucl. Phys. B499 (1997) 217, hep-th/9703040.

[25] S.S. Gubser and I.R. Klebanov, "Absorption by branes and Schwinger terms in the world volume theory," Phys. Lett. B413 (1997) 41, hep-th/9708005.

[26] G.T. Horowitz and A. Strominger, "Black strings and p-branes," Nucl. Phys. B360 (1991) 197.

[27] E. Witten, "Anti-de Sitter Space, Thermal Phase Transition, and Confinement in Gauge Theories," hep-th/9803131.

[28] M.J. Duff and J.X. Lu, "The selfdual type IIB superthreebrane," Phys. Lett. B273 (1991) 409; M.J. Duff, R. Khuri, J.X. Lu, "String solitons," Phys. Rept. 259 (1995) 213.

[29] G.W. Gibbons and P.K. Townsend, "Vacuum interpolation in supergravity via super p-branes," Phys. Rev. Lett. 71 (1993) 3754, hep-th/9307049.

[30] S.S. Gubser, I.R. Klebanov, and A.A. Tseytlin, "Coupling constant dependence in the thermodynamics of $\mathrm{N}=4$ supersymmetric YangMills theory," Nucl. Phys. B534 (1998) 202, hep-th/9805156.
[31] A. Fotopoulos, T.R. Taylor, "Comment on two loop free energy in $N=4$ supersymmetric Yang-Mills theory at finite temperature," Phys. Rev. D59 (1999) 044035, hep-th/9811224;

M. Vázquez-Mozo, "A Note on Supersymmetric Yang-Mills Thermodynamics," hep-th/9905030;

C. Kim and S.-J. Rey, "Thermodynamics of Large-N Super Yang-Mills Theory and AdS/CFT Correspondence," hep-th/9905205;

A. Nieto and M. Tytgat, "Effective field theory approach to $\mathrm{N}=4$ supersymmetric Yang-Mills at finite temperature," hep-th/9906147.

[32] I.R. Klebanov, W. Taylor IV and M. Van Raamsdonk, "Absorption of dilaton partial waves by D3-branes," hep-th/9905174.

[33] A. A. Tseytlin, "On Non-Abelian Generalisation of Born-Infeld Action in String Theory," Nucl. Phys. B501 (1997) 41, hep-th/9701125.

[34] A. Petkou and K. Skenderis, "A nonrenormalization theorem for conformal anomalies," hep-th/9906030.

[35] S. Hyun, "U-duality between Three and Higher Dimensional Black Holes," hep-th/9704005;

K. Sfetsos and K. Skenderis, "Microscopic derivation of the Bekenstein-Hawking entropy formula for non-extremal black holes," hep-th/9711138.

[36] M. Günaydin, L.J. Romans and N. Warner, "Compact and non-compact gauged supergravity in five dimensions," Nucl. Phys. B272 (1986) 598.

[37] L. Romans, "New compactifications of chiral $N=2, d=10$ supergravity," Phys. Lett. B153 (1985) 392.

[38] M.J. Duff, B.E.W. Nilsson and C.N. Pope, "Kaluza-Klein supergravity," Phys. Rep. 130 (1986) 1.

[39] J.H. Schwarz, "Covariant field equations of chiral $N=2, D=10$ supergravity," Nucl. Phys. B226 (1983) 269.

[40] H.J. Kim, L.J. Romans and P. van Nieuwenhuizen, "Mass spectrum of chiral ten dimen- 
sional $\mathcal{N}=2$ supergravity on $\mathbf{S}^{5}$," Phys. Rev. D32 (1985) 389; M. Günaydin and N. Marcus, "The spectrum of the $S^{5}$ compactification of the chiral $N=2, D=10$ supergravity and the unitary supermultiplets of $U(2,2 \mid 4)$," Class. Quant. Grav. 2 (1985) L11.

[41] J. Maldacena, "Wilson loops in large $N$ field theories," Phys. Rev. Lett. 80 (1998) 4859, hep-th/9803002; S.-J. Rey and J. Yee, "Macroscopic strings as heavy quarks in large $N$ gauge theory and anti-de Sitter supergravity", hep-th/9803001.

[42] R.R. Metsaev and A.A. Tseytlin, "Type IIB superstring action in $A d S_{5} \times S^{5}$ background," Nucl. Phys. B533 (1998) 109, hep-th/9805028; R. Kallosh and A.A. Tseytlin, "Simplifying superstring action on $A d S_{5} \times S^{5}$," J. High Energy Phys. 9810 (1988) 016, hep-th/9808088.

[43] L. Susskind and E. Witten, "The holographic bound in Anti-de Sitter space," hep-th/9805114.

[44] D.Z. Freedman, S. Mathur, A. Matusis and L. Rastelli, "Correlation functions in the $\mathrm{CFT}_{D} / \mathrm{AdS}_{D+1}$ correspondence," hep-th/9804058.

[45] I.R. Klebanov and E. Witten, "AdS/CFT correspondence and Symmetry Breaking," hep-th/9905104.

[46] P. Breitenlohner and D.Z. Freedman, "Stability in Gauged Extended Supergravity", Ann. Phys. 144 (1982) 249.

[47] V. Balasubramanian, P. Kraus and A. Lawrence, "Bulk vs. Boundary Dynamics in Anti-de Sitter Spacetime," hep-th/9805171.

[48] W. Mück and K. S. Viswanathan, "Regular and Irregular Boundary Conditions in the AdS/CFT Correspondence," hep-th/9806155.

[49] P.S. Howe and P.C. West, "Superconformal invariants and extended supersymmetry," Phys. Lett. B400 (1997) 307.

[50] S. Ferrara, C. Fronsdal and A. Zaffaroni, "On $N=8$ supergravity on $A d S_{5}$ and $N=4$ su- perconformal Yang-Mills theory," Nucl. Phys. B532 (1998) 153, hep-th/9802203.

[51] E. D’Hoker, D. Z. Freedman, S. Mathur, A. Matusis and L. Rastelli, "Graviton exchange and complete 4-point functions in the AdS/CFT correspondence, " hep-th/9903196.

[52] G. Arutyunov and S. Frolov, "Four-Point Functions of Lowest Weight CPO's in $\mathrm{N}=4 \mathrm{SYM}_{4}$ in Supergravity Approximation," hep-th/0002170.

[53] M.R. Douglas and G. Moore, "D-branes, quivers, and ALE instantons," hep-th/9603167.

[54] P. Candelas and X. de la Ossa, "Comments on Conifolds," Nucl. Phys. B342 (1990) 246.

[55] S.S. Gubser, "Einstein Manifolds and Conformal Field Theories," Phys. Rev. D59 (1999) 025006, hep-th/9807164.

[56] A. Ceresole, G. Dall'Agata, R. D'Auria, and S. Ferrara, "Spectrum of Type IIB Supergravity on $A d S_{5} \times T^{1,1}$ : Predictions On $\mathcal{N}=1$ SCFT's," hep-th/9905226. 